\title{
BMJ
}

\section{Risk of pre-eclampsia in first and subsequent pregnancies: prospective cohort study}

\author{
Sonia Hernández-Díaz, associate professor, ${ }^{1}$ Sengwee Toh, doctoral student, ${ }^{1}$ Sven Cnattingius, professor ${ }^{2}$
}

\begin{abstract}
${ }^{1}$ Department of Epidemiology, Harvard School of Public Health, 677 Huntington Ave, Boston, MA 02115, USA

${ }^{2}$ Department of Medical Epidemiology and Biostatistics and Clinical Epidemiology Unit, Department of Medicine, Karolinska Institute, S-171 77 Stockholm, Sweden

Correspondence to: S Hernández-

Díaz shernan@hsph.harvard.edu

Cite this as: BMJ 2009;338:b2255 doi:10.1136/bmj.b2255
\end{abstract}

\author{
ABSTRACT \\ Objective To investigate whether pre-eclampsia is more \\ common in first pregnancies solely because fewer \\ affected women, who presumably have a higher risk of \\ recurrence, go on to have subsequent pregnancies. \\ Design Prospective cohort study. \\ Setting Swedish Medical Birth Register.
}

Participants 763795 primiparous mothers who had their first births in Sweden, 1987-2004.

Main outcome measures Pre-eclampsia.

Results The risk of pre-eclampsia was $4.1 \%$ in the first pregnancy and $1.7 \%$ in later pregnancies overall. However, the risk was $14.7 \%$ in the second pregnancy for women who had had pre-eclampsia in their first pregnancy and $31.9 \%$ for women who had had pre-eclampsia in the previous two pregnancies. The risk for multiparous women without a history of pre-eclampsia was around $1 \%$. The incidence of pre-eclampsia associated with delivery before 34 weeks' gestation was $0.42 \%$ in primiparous women, $0.11 \%$ in multiparous women without a history of pre-eclampsia, and $6.8 \%$ and $12.5 \%$ in women who had had one or two previous pregnancies affected, respectively. The proportion of women who went on to have a further pregnancy was $4-5 \%$ lower after having a pregnancy with any pre-eclampsia but over $10 \%$ lower if pre-eclampsia was associated with very preterm delivery. The estimated risk of pre-eclampsia in parous women did not change with standardisation for pregnancy rates.

Conclusions Having pre-eclampsia in one pregnancy is a poor predictor of subsequent pregnancy but a strong predictor for recurrence of pre-eclampsia in future gestations. The lower overall risk of pre-eclampsia among parous women was not explained by fewer conceptions among women who had had pre-eclampsia in a previous gestation. Early onset pre-eclampsia might be associated with a reduced likelihood of a future pregnancy and with more recurrences than late onset pre-eclampsia when there are further pregnancies. Findings are consistent with the existence of two distinct conditions: a severe recurrent early onset type affected by chronic factors, genetic or environmental, and a milder sporadic form affected by transient factors.

\section{INTRODUCTION}

Pre-eclampsia has been defined as a disease of first pregnancies. ${ }^{1}$ The association between primiparity and pre-eclampsia is so widely accepted that it is at the core of several pathophysiological theories. For example, it has been proposed that pre-eclampsia is the consequence of a maternal immune reaction against paternal antigens expressed in the placenta and that this reaction might result in defective trophoblast invasion and subsequent placental dysfunction. The lower risk of pre-eclampsia among multiparous women has been attributed to desensitisation after exposure to paternal antigens in the placenta during previous pregnancies. ${ }^{12}$ The lower risk has also been attributed to smoother trophoblastic invasion after modification of maternal spiral arteries during the first pregnancy. ${ }^{3}$

We hypothesised that pre-eclampsia is more common in first pregnancies because women who experience pre-eclampsia during their first pregnancy, whom might have an increased risk for genetic or environmental reasons, might decide not to take further risks and have no more pregnancies. In addition, subfecundity has been associated with a higher risk of preeclampsia. ${ }^{4}$ Thus an underlying disorder might result in both pre-eclampsia in the first pregnancy and subsequent infertility.

Most previous studies included relatively small cohorts of pregnancies without prospective follow-up of women from the beginning of their reproductive history. ${ }^{15}$ We used a large population based cohort to investigate the association between parity and the risk of pre-eclampsia, before and after adjustment for potential selection bias.

\section{METHODS}

Study population

The Swedish Medical Birth Register is a population based registry that includes more than $98 \%$ of all births in Sweden. ${ }^{6}$ Starting with the first antenatal visit, normally at $8-12$ weeks' gestation, information is prospectively collected on maternal demographic data, reproductive history, pregnancy, and birth characteristics. Such information is recorded on the standardised antenatal, obstetric, and neonatal records, and copies of these records are forwarded to the birth register, where the information is computerised. The current study included only women who had their first delivery on or after 1 January 1987, with reproductive 
history followed until the end of 2004. During this period, the register recorded 763795 primiparous mothers who had a total of 1430464 deliveries (21 629 (1.5\%) were multiple gestations).

\section{Outcome definition and ascertainment}

The diagnoses are noted by a doctor at the time of discharge from the hospital, using ICD-9 and ICD-10 (international classification of diseases, 9th and 10th revisions). Pre-eclampsia is defined as a diastolic blood pressure of at least $90 \mathrm{~mm} \mathrm{Hg}$ accompanied by proteinuria $(\geq 0.3 \mathrm{~g} /$ day or $\geq 1$ on a urine dipstick) (ICD9 codes $642 \mathrm{E}-\mathrm{F}$ and ICD-10 code O14). Eclampsia is defined as the occurrence of pre-eclampsia with seizures (ICD-9 code $642 \mathrm{G}$ and ICD-10 code O15). We included pre-eclamptic and eclamptic pregnancies in our analysis. The quality of the diagnosis of preeclampsia has previously been validated: of 148 pregnancies coded as pre-eclampsia in the birth register, $137(93 \%)$ had pre-eclampsia according to the individual records. ${ }^{7}$

Early and late onset pre-eclampsia have different heritability, risk factors, clinical manifestations, and prognosis. ${ }^{8-13}$ As information on timing of onset was not available through the register, we used preterm delivery as a proxy for early onset and severity. Preeclampsia was considered "severe" when delivery occurred before 34 completed gestational weeks.

\section{Statistical analysis}

We estimated the overall cumulative incidence of preeclampsia during the first pregnancy. Among those with and without a diagnosis, we estimated the proportion of women who had a second pregnancy and the incidence of pre-eclampsia during that pregnancy. We repeated the procedure up to the fourth pregnancy. We calculated the interval between pregnancies with the difference between dates of last menstrual periods.

To compare the frequency of a second pregnancy between women with and without pre-eclampsia in their first pregnancy, we estimated the odds ratio and 95\% confidence interval for a subsequent pregnancy. Multivariate logistic regression adjusted for potential confounding factors - that is, factors associated with the risk of both pre-eclampsia and subsequent pregnancies, including maternal age, body mass index, cigarette smoking, diabetes, and multiple gestations. $^{7111415}$ To further assess the impact of multiple gestations, we conducted a subanalysis restricting the sample to singleton births. To assess the impact of time trends, we conducted a subanalysis stratified by calendar years of first pregnancy.

To quantify the contribution of any selection by history of pre-eclampsia to the difference in the incidence of pre-eclampsia between first and subsequent pregnancy, we standardised the analysis by the rates of subsequent pregnancy in women without pre-eclampsia. That is, we simulated a population where the likelihood of a subsequent pregnancy was equal among women with and without a history of pre-eclampsia. We then estimated the risk of pre-eclampsia among multiparous women in such hypothetical population.

\section{RESULTS}

The risk of pre-eclampsia in any pregnancy was 3.0\%; the risk was $4.1 \%$ in the first pregnancy and $1.7 \%$ in later pregnancies. The risk during a given pregnancy, however, was highly dependent on the history of preeclampsia (fig 1). During the second pregnancy, the risk was $14.7 \%$ for women who had developed preeclampsia in their first pregnancy and $1.1 \%$ for those who had not. During the third pregnancy, the risk was $31.9 \%$ for women who had developed pre-eclampsia in the previous two pregnancies and remained $1.1 \%$ for those without a history of pre-eclampsia. Similarly, for women with a first occurrence of pre-eclampsia in their second pregnancy, the risk was 15.9\% during the third pregnancy; and 29.0\% during the fourth when they had developed pre-eclampsia in the previous two pregnancies. Likewise, for women with a first occurrence of

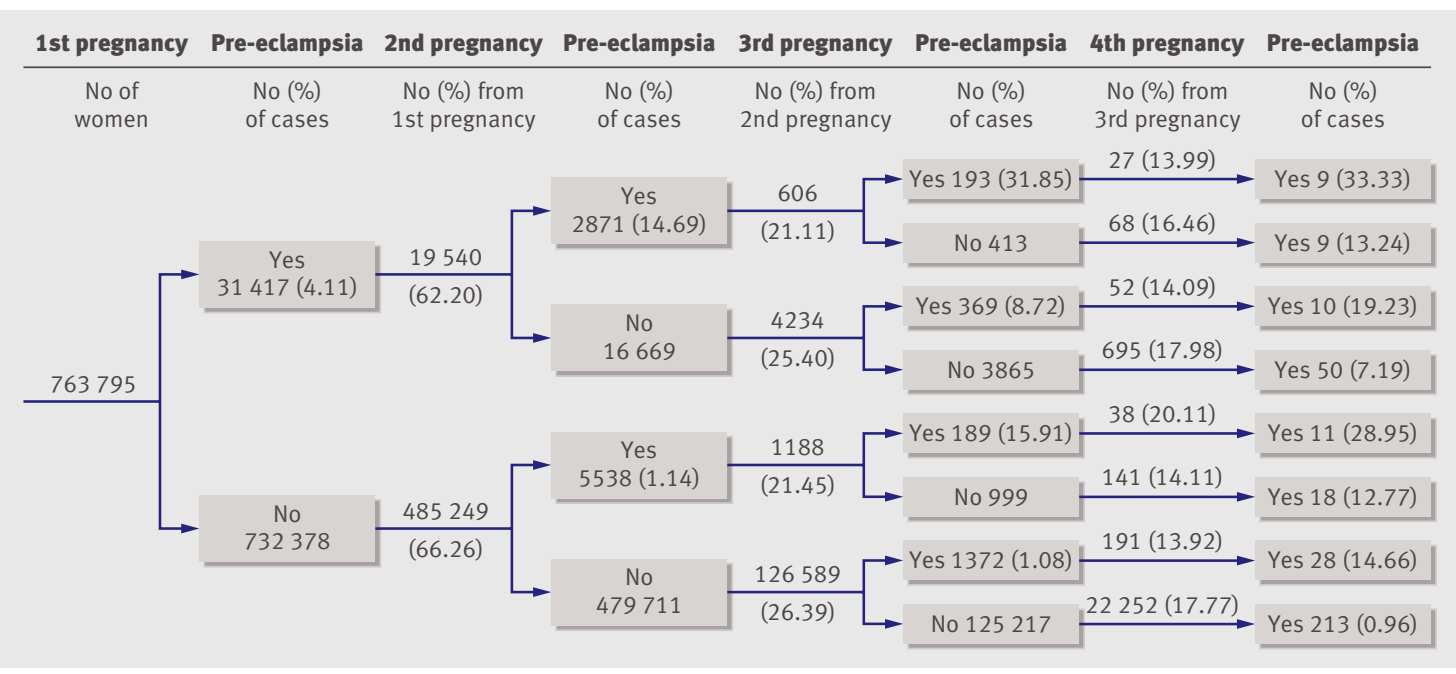

Fig 1| Risk of pre-eclampsia in given pregnancy by pregnancy order and history of pre-eclampsia, Swedish Medical Birth Register, 1987-2004 
pre-eclampsia in their third pregnancy, the risk was 14.7\% during the fourth pregnancy. Among women without pre-eclampsia in their first pregnancy, the risk of pre-eclampsia was $0.83 \%$ if they became pregnant again within two years and $2.2 \%$ if they became pregnant more than eight years after their first pregnancy; the corresponding risks were $13.1 \%$ and $15.8 \%$ for women with pre-eclampsia in their first pregnancy (fig 2).

The proportion of women with two or more pregnancies was $62.2 \%$ and $66.3 \%$ among women with and without pre-eclampsia in their first pregnancy, respectively (fig 1). The proportion of women with three or more pregnancies ranged from $21.1 \%$ to $26.4 \%$ depending on history. If women with and without pre-eclampsia in their first pregnancy had the same likelihood of another pregnancy (that is, if there were no selection of low risk women in subsequent pregnancies), the estimated incidence of pre-eclampsia among multiparous women would have been $1.70 \%$, similar to the observed $1.65 \%$.

\section{Impact of confounders}

After having a first pregnancy with pre-eclampsia, the adjusted odds ratio for having another pregnancy was 0.91 (95\% confidence interval 0.89 to 0.94 ) compared with women without pre-eclampsia in their first pregnancy. This adjusted odds ratio was closer to the null than the crude odds ratio $(0.84,0.82$ to 0.86$)$, suggesting that the observed association between pre-eclampsia and the likelihood of subsequent pregnancies was partially explained by measured confounders.

\section{Impact of multiple gestations}

The results were similar when we limited our analysis to women with a singleton birth in the first pregnancy (data not shown). Among the 12756 (1.7\%) women who had twins (or more) during their first pregnancy, $12.3 \%$ developed pre-eclampsia during that pregnancy. During the second pregnancy, the risk was $6.3 \%$ for women who had developed pre-eclampsia in their first pregnancy and $1.1 \%$ for those who had not. The proportion of women with a second pregnancy was $25.4 \%$ after having a first twin pregnancy with pre-eclampsia and $26.4 \%$ after a twin pregnancy without pre-eclampsia.

\section{Impact of temporal trends}

The risk of pre-eclampsia during first pregnancy was $3.2 \%$ in $1987-9,4.0 \%$ in $1990-4,4.8 \%$ in $1995-9$, and $4.2 \%$ in $2000-4$. Among women without pre-eclampsia in the first pregnancy, the proportion with a subsequent pregnancy by the end of follow-up was $83.5 \%$ in $1987-9,80.5 \%$ in $1990-4,73.8 \%$ in $1995-9$, and $31.0 \%$ in 2000-4. (Note the increasingly incomplete follow-up of women's reproductive life over study years.) The proportion of subsequent pregnancy among women with pre-eclampsia in first pregnancy was $3 \%$ to $5 \%$ lower within each time period.

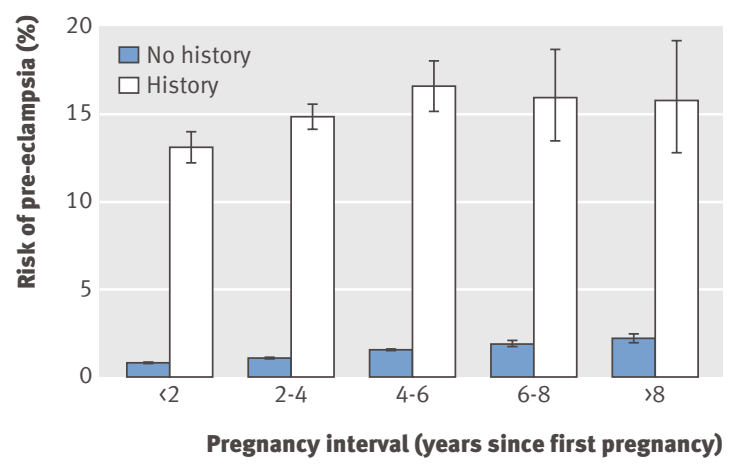

Fig 2 | Risk of pre-eclampsia in second pregnancy by years since first pregnancy and history of pre-eclampsia, Swedish Medical Birth Register, 1987-2004

\section{Impact of outcome definition}

Ten percent of primiparous women with pre-eclampsia delivered before 34 weeks of gestation. The risk of such severe pre-eclampsia was $0.42 \%$ in the first pregnancy and $0.14 \%$ in later pregnancies (fig 3). Among women who had developed severe pre-eclampsia in their first pregnancy, the risk of any pre-eclampsia was $29 \%$ in their second pregnancy; and the risk of severe pre-eclampsia was 62 times higher $(6.8 \%)$ than in women without pre-eclampsia in their first pregnancy $(0.11 \%)$. During the third pregnancy, the risk of severe pre-eclampsia was $12.5 \%$ for women who had developed this condition in the previous two pregnancies and $0.13 \%$ for those without a history of severe pre-eclampsia.

The proportion of women having a second pregnancy was $11 \%$ lower after a first pregnancy with severe pre-eclampsia than in women without this condition in their first pregnancy; the proportion of women with three or more pregnancies ranged from $13.5 \%$ to $29.2 \%$, depending on history. Despite this apparent selection of lower risk women, the estimated incidence of severe pre-eclampsia among multiparous women would have been just $0.15 \%$ (rather than $0.14 \%$ ) if women with and without severe pre-eclampsia in their first pregnancy had the same likelihood of another pregnancy. After having a first pregnancy with severe pre-eclampsia, the adjusted odds ratio of a subsequent pregnancy was 0.69 (0.64 to 0.75$)$ compared with women without the condition in their first pregnancy, which was closer to the null than the crude odds ratio $(0.63,0.59$ to 0.68$)$.

\section{DISCUSSION}

In this large prospective cohort study, the incidence of pre-eclampsia was $4.1 \%$ in primiparous women and $1.7 \%$ in parous women in Sweden between 1987 and 2004. The increased risk of pre-eclampsia in primiparous compared with multiparous women was not explained by a biological or self selection of low risk women throughout gestations. So, is pre-eclampsia really a disease of primiparous women? It depends on the history. For multiparous women, the risk was significantly different for women who had pre-eclampsia in one (around 15\%) or two (around 30\%) 
consecutive previous pregnancies than for those with no history (around 1\%). Although a longer interval between first and second pregnancies was associated with a higher incidence of pre-eclampsia, the risk for women without pre-eclampsia during their first pregnancy remained substantially lower even after eight years $(2.2 \%)$, compared with women with pre-eclampsia in their first pregnancy.

\section{Multiple gestations}

Multiple gestations were associated with an increased risk of pre-eclampsia (12\%) and with a lower recurrence of pregnancy. Yet, multiple gestations did not explain the association between primiparity and preeclampsia. Interestingly, the proportion of women with subsequent pregnancies after having twins or triplets was similar to that after two singleton births. This observation is compatible with families having a target number of children, as well as with a higher incidence of multiple gestations in subfertile couples undergoing infertility treatments. It is also worth noting that the recurrence of pre-eclampsia when the first affected pregnancy was a multiple gestation was half of that found when it was a singleton pregnancy. This finding suggests that a proportion of pre-eclampsias in multiple gestations might be caused by the fetal multiplicity itself, rather than to underlying constant maternal risk factors.

\section{Temporal trends}

The observed slight rise in the risk of pre-eclampsia over time could be caused by an actual increase in the risk of pre-eclampsia (for example, because of an increase in multiple gestations) or to changes in diagnosis and coding practices. The proportion of women having two or more pregnancies decreased over the study period, which could be partially explained by an increasing proportion of couples having a single child during the 1990s. At least part of the apparent

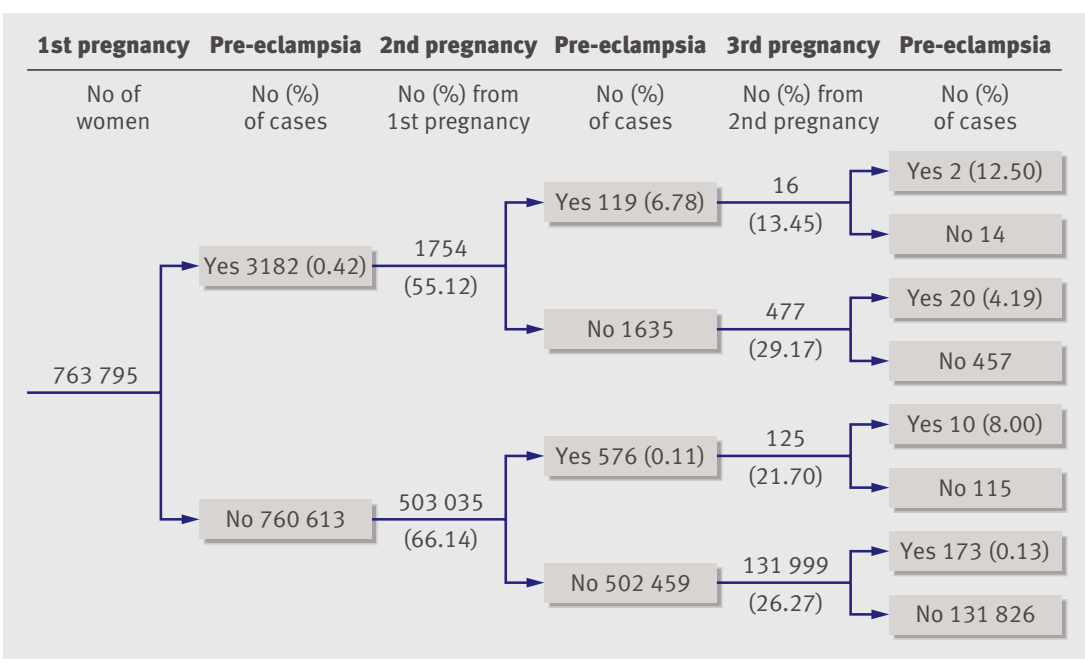

Fig 3 | Risk of severe pre-eclampsia (defined as pre-eclampsia associated with delivery before 34 weeks) in given pregnancy by pregnancy order and history of pre-eclampsia, Swedish Medical Birth Register, 1987-2004 trend, however, is because of an increasing underestimation of the incidence of subsequent pregnancies induced by the study design. By the end of 2004, when follow-up stopped, a considerable proportion of women had not completed their families, in particular those with a first pregnancy during the 2000s. Nonetheless, such trends in the recurrence of both preeclampsia and pregnancy did not explain the results. The association between pre-eclampsia and a lower incidence of subsequent pregnancies was similarly modest, and the association between parity and preeclampsia remained within each stratum of calendar years.

\section{More than one condition under the same term}

Early and late onset pre-eclampsia differ in their risk factors and prognoses. ${ }^{9-13}$ One study has shown that the earlier pre-eclampsia develops in a given pregnancy, the greater the risk that pre-eclampsia will develop in subsequent pregnancies. ${ }^{8}$ Findings from the Swedish Medical Birth Register are consistent with this. ${ }^{7}$ In the current study, a history of early onset pre-eclampsia was a stronger predictor for the recurrence of preeclampsia than late onset events, in particular for the recurrence of early onset pre-eclampsia. For preeclampsia overall, the risk of recurrence was close to 15 and 30 times higher after one or two affected pregnancies, respectively. The relative risk of recurrence after one or two pregnancies with early onset preeclampsia was 60 and 90, respectively. These observations suggest that early and late onset events might indeed differ etiologically, with early onset preeclampsia being affected by more chronic risk factors.

Results also suggest that early onset pre-eclampsia results more often in avoidance of future pregnancies, presumably because of the more severe clinical presentation compared with late onset events. The incidence of a second pregnancy was $66 \%$ in women without preeclampsia in their first pregnancy, $63 \%$ when women developed pre-eclampsia without prematurity in the first pregnancy, and 55\% if the pre-eclampsia was associated with very preterm delivery. Such selection was still insufficient to explain the association between severe pre-eclampsia and primiparity.

\section{Interpretation of findings}

Our findings support the idea of two distinct entities, one sporadic and another recurrent. We hypothesise that the sporadic type occurs in around 1\% of pregnancies and is associated with transient risk factors (such as twins), being therefore a poorer predictor of risk in future pregnancies and less predictable based on history. The recurrent type represents $50-75 \%$ of the cases in primiparous women, tends to have an early onset, is more severe, causes preterm delivery and other fetal and maternal morbidities, has more familial aggregation, and is associated with unvarying risk factors (environmental or genetic) that confer a risk of around $30 \%$ in each pregnancy in that particular group of women. Not every woman with these chronic risk factors, however, develops recurrent type pre-eclampsia, 


\section{WHAT IS ALREADY KNOWN ON THIS TOPIC}

The risk of pre-eclampsia is higher in first pregnancies (4.1\%) than in subsequent ones $(1.7 \%)$

\section{WHAT THIS STUDY ADDS}

The lower overall risk of pre-eclampsia among parous women is not explained by fewer pregnancies among women who experienced pre-eclampsia in a previous gestation

The risk of recurrence is around $15 \%$ for women who had pre-eclampsia in one previous pregnancy and around $30 \%$ when two consecutive previous pregnancies were affected

Recurrence is higher for pre-eclampsias associated with very preterm delivery

and not every woman with recurrent type pre-eclampsia in their first pregnancy has pre-eclampsia in further gestations. Moreover, with our current knowledge (and available data), we cannot distinguish sporadic from recurrent types when we diagnose pre-eclampsia in a primiparous woman. A proportion of cases in primiparous women will be sporadic and would not tend to recur.

\section{Clinical relevance}

In addition to contributing to our understanding of pre-eclampsia, our findings might influence the information women are given about the risk of pre-eclampsia in a subsequent pregnancy by providing risk estimates stratified by history. For instance, when advising women who developed pre-eclampsia in their first pregnancy and are contemplating a second pregnancy, we cannot say that their risk is low because pre-eclampsia is a "disease of nulliparity," particularly if they had an early onset event.

\section{Strengths and weaknesses of the study}

Our study is based on a large, prospective, population based cohort. The accuracy of diagnoses in the Swedish Medical Birth Register was validated, and the positive predictive values were high. ${ }^{7}$ We evaluated the effect of well known risk factors as potential confounders and effect modifiers including multiple gestations, maternal age, diabetes, body mass index, and time trends in incidence or diagnosis of preeclampsia. ${ }^{711415}$ Adjusted estimates of the effect of pre-eclampsia on the likelihood of future pregnancies were similar to crude ones, suggesting that confounding did not explain results. We were unable to directly assess the effect of timing of onset or clinical severity because data were not available in the register. Rather, we used a proxy for early onset and severity - that is, pre-eclampsia associated with delivery before 34 gestational weeks.

Further research is needed to characterise the different disorders under the classification of gestational hypertension and proteinuria. A correct diagnosis might help us to predict the risk in future pregnancies for individual women, as well as to understand the etiology and find better approaches to prevent and treat each disorder.

\section{Conclusion}

Having pre-eclampsia in one pregnancy is a poor predictor for subsequent pregnancy but a strong predictor for recurrence of pre-eclampsia in future gestations. Contrary to what we had hypothesised, the lower overall risk of pre-eclampsia among parous women was not explained by fewer conceptions among women who experienced pre-eclampsia in a previous gestation. Early onset pre-eclampsia was associated with more recurrence of pre-eclampsia and fewer subsequent pregnancies than late onset events. Findings are consistent with the existence of two distinct conditions: a severe early onset type affected by chronic factors, genetic or environmental, and a milder sporadic type affected by transient factors.

We thank Miguel Hernan for his useful comments

Contributors: All authors jointly designed the hypothesis, analysed and interpreted the data, and wrote the paper. SHD is guarantor. Funding: This research received no specific grant from any funding agency in the public, commercial, or not-for-profit sectors. The authors had full access to all the data in the study and had the final responsibility for the decision to submit for publication.

Competing interests: None declared.

Ethical approval: The study was approved by the research ethic committee at Karolinska Institute, Sweden (No 4863/2005).

1 Luo Z, An N, Xu H, Larante A, Audibert F, Fraser W. The effects and mechanisms of primiparity on the risk of pre-eclampsia: a systematic review. Paediatr Perinat Epidemiol 2007;21(suppl):36-45.

2 Saftlas AF, Levine R, Klebanoff M, Martz KL, Ewell MG, Morris CD, et al. Abortion, changed paternity, and risk of preeclampsia in nulliparous women. Am J Epidemiol 2003;157:1108-14.

3 Moore M, Redman CWG. Case-control study of severe pre-eclampsia of early onset. BM/ 1983;287:580-3.

4 Basso O, Weinberg C, Baird D, Wilcox A, Olsen J. Subfecundity as a correlate of preeclampsia: a study within the Danish National Birth Cohort. Am J Epidemiol 2003;157:195-202.

5 Duckitt K, Harrington D. Risk factors for pre-eclampsia at antenatal booking: systematic review of controlled studies. $B M$ J 2005;330:549-50.

6 Evaluation of the Swedish Medical Birth Register. Epidemiologic Centre NBoHaW, Sweden, 2008. www.socialstyrelsen.se/ Publicerat/2002/2023/2002-112-4.htm (in Swedish).

7 Ros H, Cnattingius S, Lipworth L. Comparison of risk factors for preeclampsia and gestational hypertension in a population-based cohort study. Am J Epidemiol 1998;147:1062-70.

8 Sibai BM, Mercer B, Sarinoglu C. Severe preeclampsia in the second trimester: recurrence risk and long-term prognosis. Am J Obst Gynecol 1991;165:1408-12.

9 CLASP. CLASP: a randomized trial of low-dose aspirin for the prevention and treatment of pre-eclampsia among 9364 pregnant women. Lancet 1994;343:619-29.

10 Dekker G, de Vries J, Doelitzsch P, Huijegens P, von Blomberg B, Jakobs $C$, et al. Underlying disorders associated with severe earlyonset preeclampsia. Am J Obstet Gynecol 1995;173:1042-8.

11 Myatt L, Miodovnik M. Prediction of preeclampsia. Semin Perinatol 1999;23:45-57.

12 Odegard RA, Vatten L), Nilsen ST, Salvesen KA, Austgullen R. Risk factors and clinical manifestations of pre-eclampsia. Br J Obstet Gynaecol 2000;107:1410-6.

13 Hernández-Díaz S, Werler MM, Louik C, Mitchell AA. Risk of gestational hypertension in relation to folic acid supplementation during pregnancy. Am J Epidemiol 2002;156:806-12.

14 Duckitt K, Harrington D. Risk factors for pre-eclampsia at antenatal booking: systematic review of controlled studies. $B M$ J 2005;330:565.

15 Hernández-Díaz S, Werler M, Mitchell A. Gestational hypertension in pregnancies supported by infertility treatments: role of infertility, treatments, and multiple gestations. Fertil Steril 2007;88:438-45

Accepted: 26 February 2009 